manner of thought will be fostered as the child grows older by his appreciation of the fact that his conduct based upon this mode of interpretation is found to yield practical, and desired, results.

It would thus appear that the attribution of a consciousness characteristic to other men, connected with their behavior, is not due to any knowledge that transcends experience, but is due to a quite natural interpretation of the part of that experience which relates to the behavior of others, in terms of the much more frequently observed part of that experience which relates to ourselves.

New York CitY.

Henry Rutgers Marshall.

\title{
CONSCIOUS BEHAVIOR
}

$\mathrm{M}^{\mathrm{ANY}}$ references in recent issues of this Journal give the im1 pression that when the behaviorist denies that consciousness must be regarded as an independent entity, this is equivalent to relinquishing the study of part of the subject-matter of traditional psychology. Some philosophers and psychologists seem to regard the behaviorists as animal psychologists who have availed themselves of the expedient of simply ignoring what they do not understand and then regarding this high-handed brushing aside of difficult problems as equivalent to a solution of these problems. This conception so manifestly underlies Mr. H. R. Marshall' $\mathrm{s}^{1}$ objection to behaviorism that an attempt may not be inappropriate to indicate how the most baffling problems of human conduct may be investigated without utilizing the subjective methods of traditional psychology. The behaviorist merely maintains that the biological methods used in natural science can also be applied to those phenomena which have been designated as conscious or mental.

The immediate epistemological problem is to demonstrate that the concept of consciousness may be eliminated from the descriptions and explanations of human conduct and yet include all behavior, from the simplest types of animal behavior to the most complex human adjustments. The issue is clearly stated in Mr. Marshall's article. After differentiating animal behavior into the two types of reflex or instinctive on the one hand, and highly complex behavior that is hesitant, on the other, he continues: "The biologist studies both of these types of behavior in all forms of animal life; in the higher animals and in man, and in both cases quite objectively....

"But the biological student is himself a man, and as he observes his own activities, still as part of the objective world, he discovers

1"Behavior." This JournaL, Vol. XV., No. 10, pp. 258-261. 
in them these same two types of behavior. When in regard to his own body he studies that highly complex form of behavior that is hesitant and not immediate, he finds all that he discovers in connection with his studies of this type of behavior in other animals; but in very many cases he discovers also something more. He finds not only behavior of this special type but 'conscious behavior.'

"In this observation of his own behavior the student then has not only the characteristics that yield the special sciences of neurology and biochemistry, for instance, but a quite different characteristic that yields the special science of the conscious; and this is what has always been designated as psychology."

Considered merely as a statement of fact it is not likely that any behaviorist denies anything contained in the preceding quotation. However, it is open for discussion whether the differentiation into conscious and (by implication) unconscious behavior can not be displaced by a classification and method of investigation having greater heuristic possibilities, without limiting the scope of the investigation.

What is the something more that the biologist finds when he observes his own activities?

All Subjective Phenomena is Expressed in Action.-At the outset it is necessary to agree that whatever the character of this something more may be, it must be expressed in some sort of a reaction; a written account, oral description, gesture, facial expression, pantomine or any other conventional form of representation. ${ }^{2}$ Unless the something more is expressed in action of some sort it can not become the data of science or of empirical investigation. If it is maintained that consciousness is something more than a verbal report this something more must be activity of some form; changes in respiration, vaso-motor changes, glandular or visceral secretions, but since these changes are usually expressed in verbal descriptions we shall regard the speech reaction as furnishing adequate data for the discussion in this article.

To deny that consciousness must be expressed in order to be available for science places it beyond the reach of scientific investigation and within the realm of poetry or literature.

If it is granted that consciousness must be expressed as neuromuscular phenomena, the problem becomes that of determining the neural characteristics of those expressions or activities of which consciousness is predicated.

Delimitation of the Discussion.-To simplify the analysis of these characteristics we assume a concrete situation in which there is an

2 In the interest of simplicity in presentation the single phrase "speech reaction" will be used to designate any of these forms of expression. 
observer who has reported his own movements and also introspections on his "mental states or processes" in the accepted psychological manner. We shall refer to this individual as the self observer. In addition he is supposed to be under the observation of another person whom we shall call the outside observer. It is further supposed that each observer has prepared a report describing that phase of the concrete situation in which he participated. A comparison of the two reports should reveal the characteristics of what is traditionally known as conscious behavior, in so far as it is a part of our concrete situation.

Objective Behavior.-An inspection of the observers' reports reveals many reactions that are similar. For instance in the self observer's report is found, "My hand moved before my eyes," while in the outside observer's report is found, "His hand moved before his eyes." Both of these items represent coordinated contractions of the muscles used in writing. The light reflected from the hand of the self observer acts upon his own eyes and also upon those of the outside observer. A single stimulus source thus results in two sets of neural processes, each leading to independent but similar contractions of the muscles of the writing hand of each observer, in the coordinated manner which we describe as writing, the character of which (choice of words, grammatical structure, etc.) will depend upon what language habits have been acquired. All of this may be regarded as solely neural or biological in character. It is of no scientific advantage to claim that these speech reactions were accompanied by mental processes, since if no reactions had been made, no mental states could ever have been inferred. Objective behavior then can be regarded as that type of behavior in which the sensorimotor conditions are similar (not necessarily identical) for all those individuals who may be regarded as participating in the observation.

Sensory Subjective Behavior.-The self observer in addition to reporting, "My hand moved before my eyes," may also report, "I had kinesthetic sensations localized in the muscles of the forearm and shoulders; some kind of sensations which seemed to be localized in the eyes as I tried to follow the movement; strain sensations in the back and neck." The number of such observations, traditionally designated as subjective or introspective, depends largely upon the complexity of the reaction, its duration, and upon skill in discriminating obscure and weak stimuli.

Regarding these reactions as due to the stimulation of kinesthetic sense organs located in the self observer's arms, shoulder, back and eye muscles, it is clear that he has a source of stimulation not available to the outside observer from whom we should not expect a simi- 
lar reaction since no corresponding sense organs in his own body are being stimulated.

Mr. Marshall supports his contention that consciousness must be regarded as a factor in behavior by referring to the practise of the physician, "who constantly treats of the conscious states of his patients as symptomatic of special forms of organic behavior." This only means that the patient may react to obscure stimuli within his own body which, of course, can not act upon the sense organs of the physician. In other words the physician's reactions are limited mainly to visual and auditory impressions while the patient may have additional organic, kinesthetic and cutaneous impressions which do not act upon the physician's sensorium.

The reason the physician asks the patient to react to the obscure and personal stimuli is because no better way of making the diagnosis is available. Where it is possible to substitute the physician's sense organs for those of the patient it is invariably done. To illustrate: The dentist has two ways of determining whether the root of a tooth is abscessed; (1) by tapping it in various ways, pressing on the gums and asking the patient how it "feels." The "feeling" of the patient is of course due to the stimulation of the various pain and tactual sense organs which are stimulated by the tapping or pressing. This is the subjective method of diagnosis. The other method, (2) is to take an X-ray photograph of the teeth and gums. In this method the dentist substitutes his own sense organs (vision) for the pain and tactual receptors of the patient. In doubtful cases both methods are used, but where the two methods give contradictory results it is the $\mathrm{X}$-ray which is regarded as authoritative, not the subjective states of the patient.

It must be remembered that the terms subjective and objective are social distinctions. A single individual's reactions are all of one sort. Either all subjective, or all objective, or neither, depending upon what metaphysical point of view is taken. From the anatomical and physiological standpoints this distinction only designates the empirical fact that we may react to stimuli arising within our own bodies or to stimuli arising outside of the body. Those stimuli arising within our own bodies do not act on corresponding sense organs in other persons, whereas the stimuli arising outside the body may act on corresponding sense organs in any number of individuals. It is to be expected that these marked differences in stimulus conditions will result in marked differences in the reactions. Subjective and objective then, from the biological standpoint are merely terms that indirectly designate this difference in the termini of our reaction mechanisms. 
Imaginal Subjective Behavior.-In addition to reacting to sensory stimulations within his own body the self observer may also report what is known as imagery, affective states, cognitive conditions, etc., as for instance, "The movement of my hand reminds me of a semaphore action which I saw in a railway station at the end of an unpleasant trip." The immediate sensory factors in this type of observation are relatively insignificant as compared with the central neural interconnections and the extent to which the nervous system has been modified by past function. The outside observer's report may also show this effect as when he reports, "The movement of the arm was made in a very characteristic fashion; my grandfather frequently made such a movement when brushing a fly from his nose; I wonder why I happened to think of it at this time?" The two reports do not agree because the neural processes pass over two different nervous systems each one of which has been independently modified by earlier function.

When neural processes which do not correspond in their sensorimotor configuration for both observers, are strong enough to result in motor contradictions such as those assumed in this particular situation, we may speak of introspection, the characteristic of which is the use in a professional manner of such terms as, sensations, images, affections, cognition, perception, memory, etc., in our speech reactions. These terms may be used to describe our actions in a very consistent manner without referring at all to neurology so long as we wish merely to classify the reactions. When however we wish to determine the genesis of a particular mode of behavior, we are obliged to study the antecedent neural conditions.

Imaginal Subjective Incipient Behavior.-In our illustration we have assumed that the observers have actually reacted (written out their imagery). This assumes that the neural processes which get their present configuration from previous functioning are strong enough to result in the motor contractions necessary to write out a report. We should expect that frequently such processes are not strong enough to result in reactions which can be detected and inspected by an outside observer, but from other observations it has been found that actions very often do result, especially when we consider subvocal reactions.

This type of neural activity may be designated as thinking and in this sense thinking is only neuro-muscular activity which gets its character from past neural function but at the moment is not strong enough to result in speech reactions. It may become strong enough at any moment, as when an observer begins to "talk to himself" or "thinks out loud." The sense organs that are stimulated may be 
obscure kinesthetic or organic receptors in the body of the self observer, or a part of the neural flux directly involved in the overt reaction of the moment, may take its course to those motor points which actually did contract at some previous time.

When reacting to any situation the spread of the neural flux over the nervous system is always wider than necessary to react in a socially adequate manner. Supplementary speech reactions, respiratory and vaso-motor changes of no significance for the social adequacy of a reaction may and usually do occur. Under ordinary conditions we ignore them because the character of these supplementary reactions varies so much from individual to individual and for the same individual at different times that uniformity can scarcely be said to exist.

The spontaneous or conscious character of our thought processes is an illusion due to the fact that the actual neural conditions involved are obscure and can not be definitely referred to the immediate sensori-motor conditions.

Conscious Behavior.-The term conscious characterising actions is regarded by the behaviorist as merely a vague and ambiguous expression referring to undefined combinations of objective, sensory subjective, imaginal subjective, and imaginal subjective incipient behavior. $^{3}$ In another article ${ }^{4}$ the writer has attempted to give the term a definite meaning by restricting its use to those observations in which the terminology of traditional psychology is used in a descriptive sense.

The behaviorist regards all behavior as neuro-muscular action whether these actions are the ones by which bodily adjustments are made or whether they consist of the complex speech reactions whose final result is the statement of a natural law or the publication of a treatise on metaphysics. The problem of consciousness for the behaviorists is merely that of agreeing among themselves as to what types of action it will be most convenient to designate by this term. So far they have agreed that the most convenient thing is not to use the term at all. If those who insist upon the heuristic value of subjective descriptions or upon the existential character of consciousness aside from its neuro-muscular expressions will indicate clearly what they mean by the term consciousness, perhaps the behaviorist will

3 This classification is to be reyarded as representing a transition stage between the terminology of traditional psychology and that of behaviorism. It has value only as a concise abbreviation of the subject-matter discussed in this article. The writer is aware that its dualistic character will not make it acceptable either for traditional psychologists or for the behaviorists.

4"Relation between Functional and Behavior Psychology," Psychol. Rev., Vol. XXV., September, 1917, pp. 360-366. 
then be able to place it somewhere within his own categories. It is very certain, however, that defining consciousness as the something more that accompanies certain types of action is hardly enough to begin negotiations.

Critics of behaviorism do not recognize clearly enough that the term consciousness varies in its meaning with almost every person who uses it. There is no generally accepted definition or description, and the fact that psychologists and philosophers have been unable to reach an agreement is one of the conditions which precipitated behaviorism. All human conduct, aspirations, hopes and endeavors, express themselves in actions of some kind or other. Whatever the specific character of these actions may be they can not exceed the limits set by (1) the type of nervous system that has been inherited; (2) the way in which past neural function has modified it; and (3) the character of the stimuli which are immediately effective. Since these factors are never the same for any two individuals it may be useful to designate those reactions which express this difference as consciousness or mentality. However, it is the traditional psychologist who should formulate the problem of consciousness or mentality-not the behaviorist. The success of behavior methods will not depend on how they react to the problem of consciousness; they succeed or fail according as they do or do not further the general welfare of society.

Epistemologically the behaviorist supports the monistic principle that all phenomena can be reduced to action. He regards the interpolation of some hypothetical conscious principles as unnecessary.

The Illusory Spontaneity of the Speech Reaction.-Much confusion arises because the speech reaction is regarded as falling outside the laws of neuro-muscular activity that govern bodily movements (arms, legs, head, etc.). Speech is regarded as something spontaneous, that while we may recognize bodily reactions as neuromuscular in character our speech is free; we can say what we choose, argue either for or against a proposition if we wish, or remain silent when this suits our fancy. The behaviorist regards speech just as much a neuro-muscular adaptation to an environment and just as inevitable as the withdrawal of the finger from a hot stove. The significant thing about the speech reaction is that it may be either the adequate reaction to a situation, or it may be the adequate stimulus for either another speech reaction or some bodily reaction. ${ }^{5}$ Speech may thus function either as a stimulus or as a reaction.

Another reason for differentiating speech from other forms of ac-

5 This dual character of the speech mechanism has been developed by Max. F. Meyer, Fundamental Laws of Human Behavior, 1911, pp. 211-226. 
tivity is that its protective or nutritive function is indirect. The word "potato" does not have the food value of the real potato, nor will the mere pronunciation of the words, "Look out" prevent the falling brick from striking the pedestrian. Furthermore, the process of learning to make the proper speech reactions in our professional and social activities covers such a long period of time that the neural conditions involved in the learning are gradually changing. This tends to obscure the source of our speech reactions and as a consequence we do not think of them as sensori-motor adjustments to our environment of the same general type as those actions which contribute more obviously toward nutrition or protection.

Illustration of the Social Significance of the Speech Reactions. ${ }^{6}$ It is usually maintained that the high cost of living is due to the war. In investigating the situation it was found that the population of the United States during the ten years preceding the war increased twenty per cent., while food production increased only ten per cent. As a result of this investigation the following verbal statement was formulated: "The high cost of food is not entirely due to the war but is partly due to the fact that the population is increasing more rapidly than the food supply." This statement may be regarded as the adequate speech reaction of the investigator to the visual stimuli of certain records and statistics acting on his nervous system which has been variously and specifically modified by past stimulation and function. To determine just how all these factors interact to produce the speech reaction which is quoted is of course a difficult task, but a strictly empirical one, the analysis of which must consider the inheritance, training and life conditions of the investigator.

The reaction, however, may also be regarded as a stimulus which, acting on the eyes of some responsible person in the Department of Agriculture, leads to another speech reaction in the form of verbal orders from the Department to the various experiment stations and field representatives, requesting that every energy be expended to increase food production at least ten per cent. Suppose that all this comes to pass and that we have an actual increase in the food supply and a reduction in the cost of food. We can then say that the original speech reaction is very valuable and that it should be conserved for future needs. It may then become incorporated in our text-books of sociology and agriculture. The value of this particular speech reaction lies solely in the fact that it may be used as a stimulus under the proper conditions, and when so used the ultimate result is an increased food supply.

6 The illustration here used is not original with the writer. It has, however, been so "manifestly adapted" to the present needs that it is difficult to assign proper credit. 
Such a speech reaction is usually called a law, theory, hypothesis, working plan, and by reason of the fact that it is an effective stimulus to produce socially valuable actions on the part of individuals or communities it is incorporated in some phase of our educational system. The formulation of the original speech reaction is, however, a neuro-muscular phenomenon of the vocal mechanism or writing hand just as much as the more obvious skilled activity of designing or perfecting scientific apparatus.

To say that the speech reaction is the result of reasoning, logic, imagination, is merely to classify it according to properties which were at one time regarded as independent entities (faculty of reasoning, intelligence, imagination). As the scientific inadequacy of the faculty concept was demonstrated the number of faculties decreased until now they are supposed to have disappeared. The classifications, however, still remain and of course may still be used to classify our reactions, but it must be remembered that the original justification for their development (the faculty concept) no longer exists. The types of classification used in successful scientific practise to-day are those in which the principles of evolution, phylogeny, and ontogeny are predominant. Faculties or entities do not have an evolutionary, phylogenetic or ontogenic history and this fact immediately places them beyond the methods of natural science. At any rate the classification of our reactions according to the methods of traditional psychology leaves us in ignorance of the antecedent neural or biological conditions necessary to formulate other or still more valuable speech reactions.

To ask the investigator who formulated the valuable law which is quoted, to introspect on the conscious states which seemed to him to have some relation to the law, merely brings out how he might have reacted in addition to formulating the law. That is, in addition to reacting to a complex statistical situation by forming the statement, "To maintain existing economic and social conditions, the ratio between the percentage increase in population and the percentage increase in food supply must approach unity," the investigator might also have reacted by saying, "I have a visual image of a sheaf of wheat; an auditory image of the words, ten over ten equals one; a visual image of twelve people standing around a table upon which only eleven places are laid; many kinesthetic and organic sensations; fragmentary imagery of the syllogism, 'All men are mortal, Socrates is a man, Socrates is mortal.' ", These supplementary speech reactions tell us something no doubt, but the essential conditions leading to the formulation of a valuable speech reaction are so much a matter of inheritance and training, are so much a function of the whole life 
history of the individual, that the few supplementary reactions which can be induced on the occasion of its occurrence, interfere with its formulation rather than throw light upon its origin.

The behaviorist maintains that since a valuable scientific law, discovery or invention is sensori-motor phenomena, better laws, greater discoveries may be expected from the study of the biological evolution of the sensori-motor phenomena than from a study of the supplementary phenomena occurring with it.

\section{Summary}

The behaviorists maintain that the natural science methods used in biology are also adequate to investigate the phenomena that traditional psychology designates as conscious or subjective.

If subjective phenomena is to be investigated it must be expressed in neuro-muscular functions of some kind. To maintain that consciousness is something more than neuro-muscular phenomena can not be demonstrated and hence is beyond the technique of current scientific methods.

Objective behavior is that type of behavior in which the neural conditions are such that the same stimulus source acts upon corresponding sense organs for all the individuals participating in the observation. Similarity in the reactions is the test of objective behavior.

Sensory subjective behavior is that type of behavior in which the self observer is reacting to a source of stimulation (usually organic or kinesthetic) located in his own body and which stimuli can not act on corresponding sense organs in the body of the outside observer.

Imaginal subjective behavior expresses the effect of neural modifications which are due to previous functioning of the nervous system.

Imaginal subjective incipient behavior (thinking) derives its character from previous neural functioning, but at the moment under consideration the neural flux is not strong enough to result in speech reactions.

Conscious behavior may be used as a term to designate the differences between the reactions of individuals. In the absence of any considerable uniformity among traditional psychologists as to the meaning of the term consciousness, the behaviorist is justified in delaying its inclusion into his own categories until a greater degree of agreement has been established.

Much of the objection to behaviorism is due to the implicit assumption that speech reactions are spontaneous activities, instead of neuro-muscular adjustments indirectly contributing to individ- 
ual survival in the same manner as nutritive or protective reactions.

The speech reactions which are valuable for society are those which may function as effective stimuli in the further development of the physical and social resources of the community.

Since speech reactions may be regarded as sensori-motor phenomena, the direct study of their antecedent neural and general biological conditions is of greater scientific advantage than the study of concomitant or supplementary (conscious) phenomena giving only fragmentary and highly variable indices of the essential antecedents.

The traditional psychological classifications still convey at least an implicit faculty reference to many psychologists and philosophers. So difficult is it to disregard this assumption and the "specially created" attitude toward complex human behavior, that some psychologists (the behaviorists) prefer to substitute natural science concepts in which the principles of evolution, phylogeny and ontogeny are explicitly regarded as underlying their investigations.

Ohio State University.

\section{REVIEWS AND ABSTRACTS OF LITERATURE}

The Picture Completion Test. Rudolf Pintner and Margaret M. ANderson. Baltimore: Warwick and York. 1917. Pp. 101.

In this monograph the authors present a standardization of Healy's Pictorial Completion Test, which was introduced in the Psychological Review in 1914. Since that date the test has become familiar in psychological clinics. It was devised with the intention of making available a test which would involve the Ebbinghaus Completion Method, and would at the same time eliminate the factor of language. It consists of a picture, significant missing elements of which are to be supplied by choice among many alternatives pro. vided.

Hitherto the test could be given only for the purpose of gaining a general idea of the subject's mentality, and, as is the case with all unstandardized or partially standardized tests, the results were of problematical value. The authors of the present work have thus made a very useful contribution to the equipment of the psychological clinic, by determining and presenting norms of performance for every age, from five years to adult years, inclusive. In the absence of any statement to the contrary, it is probably permissible to assume that age is counted from birthday to birthday, a five-year-old, for example, being counted as five years old from his fifth birthday to 